\title{
Anti-IL-8 Monoclonal Antibody HuMax-IL8
}

National Cancer Institute

\section{Source}

National Cancer Institute. Anti-IL-8 Monoclonal Antibody HuMax-IL8. NCI Thesaurus.

Code C124227.

A human monoclonal antibody against the pro-inflammatory mediator interleukin-8 (IL-8;

CXCL8), with potential antineoplastic activities. Upon administration, HuMax-IL8 directly binds to IL-8, thereby inhibiting the binding of IL-8 to its receptors CXCR1 and CXCR2. This inhibits activation of IL-8-mediated signaling transduction pathways, which decreases proliferation of susceptible tumor cells. Also, HuMax-IL8 effectively blocks binding of IL-8 to neutrophils and inhibits neutrophil activation and recruitment towards sites of inflammation, which reduces inflammation. IL-8, a member of the CXC chemokine family, is upregulated in a variety of cancer cell types and inflammatory diseases; it plays a key role in tumor cell proliferation, endothelial cell proliferation, and cancer stem cell (CSC) renewal. 